\title{
La persistencia como fortaleza de carácter presente en estudiantes de licenciatura del centro de actualización del magisterio de Durango, México
}

\section{Persistence as a strength of character present in undergraduate students at the Durango Teacher Training Center, Mexico}

Miguel Ángel Muñoz López

Centro de Actualización del Magisterio, México

Flavio Ortega Muñoz

Centro de Actualización del Magisterio, México

Edgar Jarib Castro Luna

Centro de Actualización del Magisterio, México

Diana María Espinosa Sánchez

Centro de Actualización del Magisterio, México

Autor por correspondencia: miguelarcangel@comunidad.unam.mx, flavioaguilucho@hotmail.com, jarib15@hotmail.com, dianaespin@ hotmail.com

Fecha de recepción: 25 de Febrero de 2018 - Fecha de aceptación: 25 de Junio de 2018

Resumen: Los estudiantes de la Licenciatura en Educación Secundaria, con Especialidad en Formación Cívica y Ética, en modalidad mixta, del Centro de Actualización del Magisterio, afrontan dificultades exclusivas de la modalidad, como son: carencias económicas, períodos de descanso mínimos, duración extensa de la carrera, entre otras; con el fin de conocer sus Fortalezas de carácter, se realizó una investigación sobre el rasgo de Persistencia (VIA Institute on Character, 2017) en el carácter, esto dentro del contexto de la Psicología Positiva. El instrumento empleado para la recogida de datos fue el Inventario VIA-240. Los datos obtenidos en la investigación arrojaron que los estudiantes presentan un nivel de Persistencia alto.

Palabras Claves: persistencia; fortalezas de carácter; estudiantes de licenciatura; cam; psicología positiva

Abstract: The students of the Bachelor's Degree in Secondary Education, with Specialization in Civic Education and Ethics, in mixed modality, of the Centro de Actualización del Magisterio, face exclusive difficulties of the modality, such as: economic deficiencies, minimum rest periods, extended duration of the Career, among others; in order to know their Strengths of Character, a research was made on the trait of Perseverance (VIA, 2017) in character, this within the context of Positive Psychology. The instrument used for data collection was the VIA-240 Inventory. The data obtained in the research showed that the students present a high level of Persistence.

Key Words: persistence; strengths of character; bachelor's students; cam; positive psychology 


\section{Introducción}

En la Licenciatura en Educación Secundaria, con Especialidad en Formación Cívica y Ética, en modalidad mixta, del Centro de Actualización del Magisterio (CAM); la mayoría de los estudiantes son docentes frente a grupo, dependientes de la Secretaría de Educación Pública (SEP) o del Consejo Nacional de Fomento Educativo (CONAFE) y asumen la carrera docente como una profesión, por ello han iniciado un proceso de formación, trasladándose generalmente desde las comunidades remotas donde trabajan, experimentando al mismo tiempo, las necesidades de las familias que sostienen, con un ingreso económico neto regularmente bajo.

Este proceso de formación requiere un traslado periódico cada quince días a la capital del estado de Durango para estudiar de forma presencial los fines de semana, además de cursos intensivos sin descanso de lunes a domingo en periodos vacacionales, y una vez que terminan estos, los estudiantes deben regresar a su lugar de trabajo. La duración de la carrera es de 12 semestres, es decir, 6 años con el mínimo de vacaciones o días libres.

El estudiante de licenciatura en formato mixto es una persona que, afronta pues, muchas dificultades para terminar su carrera, por lo cual se realiza este estudio, para medir las cualidades que le permiten superar ese gran reto de estudiar y trabajar con poco o nada de descanso durante seis años; pues a pesar de esto, los estudiantes de la Licenciatura de Educación Secundaria, modalidad mixta, se mantienen matriculados, lo que da orientación para sugerir que, la Perseverancia podría ser una de las Fortalezas que comparte esta población estudiantil.

Ahora bien, con relación a la psicología positiva, Martin Seligman (como se citó en Contreras y Esguerra, 2006) la define como "el estudio científico de las experiencias positivas, los rasgos individuales positivos, las instituciones que facilitan su desarrollo y los programas que ayudan a mejorar la calidad de vida de los individuos, mientras previene o reduce la incidencia de la psicopatología" (p. 311). De igual manera, la describe como el estudio de las fortalezas humanas, así como de las virtudes. El autor sostiene que la psicología no comprende solamente el estudio de la enfermedad, la debilidad y el daño, sino también el estudio de los aspectos positivos del ser humano (Seligman y Csikszentmihalyi, 2010). Al mismo tiempo, redefine la idea hacia la mejora en el entendimiento de rasgos positivos de los jóvenes y, en sí de todas las personas, con el fin de mejorar la calidad de vida de las mismas.

En este mismo sentido y haciendo referencia en específico a las Fortalezas de Carácter presentes en la psicología positiva, y de acuerdo con Park, Peterson y Seligman (2006), así como a un estudio realizado en América Latina sobre las relaciones entre fortalezas del carácter y satisfacción con la vida (Porto y da Fonseca, 2016), las Fortalezas de Carácter son:

Características positivas reflejadas en pensamientos, sentimientos y comportamientos que pueden contribuir al buen desarrollo de los individuos, estas pueden ser clasificadas en 24: amor, amor por el aprendizaje, apreciación de lo bello, autenticidad, autorregulación, bondad, civismo, creatividad, curiosidad, esperanza, espiritualidad, imparcialidad, inteligencia social, gratitud, humor, liderazgo, modestia, pensamiento crítico, perdón, perseverancia, prudencia, sensatez, valentía, y vitalidad (p. 91) . 
Estas 24 Fortalezas de Carácter se vinculan a seis virtudes, las cuales fueron propuestas por Peterson y Seligman (2004) y se muestran clasificadas en la tabla 1.

Tabla 1. Clasificación de las 6 virtudes y 24 Fortalezas

\begin{tabular}{cccccc}
\hline Coraje & Justicia & Humanidad & Sabiduría & Templanza & Trascendencia \\
\hline Valentía & Liderazgo & Amor & Perspectiva & Humildad & Gratitud \\
Persistencia & Ciudadanía & Bondad & $\begin{array}{c}\text { Apertura } \\
\text { mental } \\
\text { Clemencia }\end{array}$ & $\begin{array}{c}\text { Apreciación de la } \\
\text { belleza }\end{array}$ \\
Integridad & Imparcialidad & $\begin{array}{c}\text { Inteligencia } \\
\text { social }\end{array}$ & $\begin{array}{c}\text { Amor por el } \\
\text { saber } \\
\text { Curiosidad } \\
\text { Creatividad }\end{array}$ & Autorregulación & $\begin{array}{c}\text { Humor } \\
\text { Vitalidad }\end{array}$ \\
& & & & Espiritualidad \\
\hline
\end{tabular}

En síntesis, las 24 Fortalezas de Carácter antes citadas fueron agrupadas en seis virtudes; en primera instancia el coraje, que está compuesta por la valentía (VIA Institute on Character, 2017), la Persistencia, la integridad y la vitalidad. La valentía es la disposición a actuar de forma voluntaria, quizás con miedo, en presencia de una circunstancia peligrosa, donde los riesgos importantes son evaluados razonablemente y sus consecuencias son aceptadas en pos de un beneficio personal o grupal; reconociendo que este bien podría no alcanzarse (Cosentino, 2010; Calderón, Forés y Gustems, 2016) .

Calderón, Gustems y Forés (2016) encontraron en un grupo de 30 estudiantes de entre 22 y 47 años, que los varones tenían entre las Fortalezas de Carácter menos desarrolladas la vitalidad; a pesar de que los planes docentes del grado de Educación Social se articulan en función a las fortalezas que engloban principalmente las virtudes de sabiduría, coraje, humanidad y justicia.

Martín-Moreno (2015) identificó que la Fortaleza Perseverancia/laboriosidad es una de las más relacionadas con el rendimiento académico de los estudiantes universitarios; lo que es consistente con el estudio realizado por Saldaña y colaboradores en 2014, con estudiantes de la Universidad de Barcelona, que las Fortalezas de Carácter estaban relacionadas con el rendimiento académico. Los resultados muestran que la honestidad, la Persistencia y la vitalidad fueron las tres fortalezas más significativas.

La Persistencia o Perseverancia, por su parte, es mantenerse constante en la prosecución de lo comenzado, en una actitud o en una opinión (Real Academia Española, 2015), es decir, la continuación voluntaria de un comportamiento activo dirigido a una meta a pesar de los obstáculos, dificultades o desalientos a fin de terminar lo que uno inició, acompañada con un sentimiento de satisfacción al terminar la tarea (Peterson, Christopher \& Stephens, John \& Lee, Fiona \& Seligman, Martin \& Park, Nansook, 2009). Para esta investigación nos centramos en analizar la Fortaleza Persistencia, manifestada en los estudiantes de Licenciatura en Educación Secundaria, con Especialidad en Formación Cívica y Ética, en modalidad mixta, del CAM del estado de Durango, México. El estudio de la Persistencia como Fortaleza de Carácter, se concreta en los siguientes objetivos:

- Determinar el nivel de Persistencia como Fortaleza de Carácter, que presentan los estudiantes de Licenciatura en Educación Secundaria del Centro de Actualización del Magisterio (CAM). 
- Establecer en qué índices empíricos tienen un mayor nivel de Persistencia los estudiantes de Licenciatura en Educación Secundaria del CAM.

- Identificar en qué ítems presentan un menor nivel de Persistencia los estudiantes de Licenciatura en Educación Secundaria del CAM.

\section{Método}

Para esta investigación se analizaron los datos de 27 estudiantes de la Licenciatura en Educación Secundaria, con Especialidad en Formación Cívica y Ética, en modalidad mixta, del CAM; no se hizo muestreo, pues se realizó la prueba al total de estudiantes. Los datos se obtuvieron a través de una prueba de auto informe llamada VIA-240, que mide las Fortalezas de Carácter; los datos obtenidos se capturaron en el software SPSS para su estudio estadístico.

\section{Sujetos}

Participaron 27 estudiantes de 5o, 7o, 9o y 11 o semestres de la Licenciatura en Educación Secundaria, con Especialidad en Formación Cívica y Ética, en modalidad mixta, del CAM; de los cuales 17 eran mujeres y 10 hombres, entre los 23 y 46 años. La prueba se aplicó en el salón de clases de cada grupo durante aproximadamente dos horas, los estudiantes respondieron los instrumentos empleando lápiz y papel.

\section{Materiales}

Para la aplicación del inventario se usó un cuestionario de autoinforme, el VIA-240, que consta de 240 reactivos con cinco opciones de respuesta tipo likert: 1. Muy diferente a mí, 2. Algo diferente a mí, 3. Neutro, 4. Algo parecido a mí, 5. Muy parecido a mí. En un estudio de Park, Peterson y Seligman (2006) que contempla 54 países, entre ellos México, se encontró que el instrumento VIA-240 tiene una consistencia interna aceptable y confiabilidad test-retest; también tiene de moderados a crecientes niveles de validez psicométrica, lo que significa que correlaciona razonablemente bien con los constructos que se esperan sean medidos y no se correlaciona con los que no deben ser medidos como la conveniencia social; en términos de validez predictiva, sus estudios demostraron niveles moderados y aceptables.

\section{Procedimiento}

Se acudió a las aulas de cada grupo con permiso previo del docente a cargo, para aplicar la prueba de auto informe. Una vez realizada la aplicación, se capturó la información obtenida en una base de datos usando el software SPSS (IBM Corp., 2013), dentro del mismo programa se realizaron las pruebas estadísticas necesarias, cuyos resultados se muestran en la siguiente sección.

\section{Resultados}

La media general obtenida en la aplicación del Inventario VIA-240 a los Estudiantes de Licenciatura en Educación Secundaria, con Especialidad en Formación Cívica y Ética, en modalidad mixta, del Centro de Actualización del Magisterio (CAM), respecto a la Fortaleza de 
Carácter Persistencia, fue 3.99. Si interpretamos este resultado con un baremo de tres valores (de 0 a 1.66, bajo; de 1.67 a 3.33, medio; y de 3.34 a 5, alto), podemos afirmar que los estudiantes encuestados presentan un alto nivel de Persistencia.

Los índices empíricos que presentan mayor nivel de Persistencia son: a) Cuando obtengo lo que quiero, se debe a que trabajo duro para alcanzarlo $(\mathrm{X}=4.56 ; \mathrm{s}=0.506)$; b) No soy de los/las que se dan por vencidos(as) $(\mathrm{X}=4.22 ; \mathrm{s}=0.934) ; \mathrm{y}$ c) Soy una persona orientada hacia las metas $(\mathrm{X}=4.19 ; \mathrm{s}=0.622)$. Los ítems en los que los estudiantes de Licenciatura en Educación Secundaria presentan un menor nivel de Persistencia son: a) Soy una persona muy disciplinada $(\mathrm{X}=3.52 ; \mathrm{s}=0.849)$; b) Nunca abordo las cosas de manera indecisa $(\mathrm{X}=3.74 ; \mathrm{s}=$ $0.712)$; у c) Yo siempre continúo con una tarea hasta terminarla $(\mathrm{X}=3.93$; $\mathrm{s}=0.616)$.

De manera particular, en el semestre quinto de dicha licenciatura se observa en los alumnos de género masculino una dificultad por alcanzar las metas que se proponen $(X=3.33$; $\mathrm{s}$ $=1.155)$ y su percepción de tener poca disciplina $(X=3.67 ; \mathrm{s}=1.155)$; por otro lado, la media de quienes aseguran revisar la factibilidad de sus metas, para no dejarlas posteriormente en su implementación, es relativamente baja $(X=2.67 ; \mathrm{s}=2.517)$.

De forma conjunta todos los integrantes de séptimo semestre se imponen metas y disfrutan en completarlas; si están indecisos, no abordan los retos $(X=3.80 ; \mathrm{s}=0.632)$; perciben deficiencias en la puntualidad $(X=4.00 ; s=1.00)$ y la disciplina $(X=3.60 ; 0.699)$. En el género femenino existe una coincidencia entre medias y la distribución de datos de los ítems $(X=4.00$; $\mathrm{s}=1.00$ ), en los reactivos referidos a perseverar y concluir metas, y la satisfacción que produce la tarea terminada. Los hombres evitan involucrarse en proyectos sin revisar su factibilidad.

El grupo de noveno semestre está compuesto completamente por mujeres, quienes se perciben como personas orientadas a metas $(X=4.00 ; \mathrm{s}=1.00)$; estas las realizan en tiempo $(\mathrm{X}$ $=4.00 ; \mathrm{s}=1.00)$ y verifican la factibilidad de las estrategias para alcanzarlas $(\mathrm{X}=4.00 ; \mathrm{s}=$ 0.707), y finalmente, sienten satisfacción al lograrlo $(X=4.40 ; \mathrm{s}=0.548)$; no obstante, su percepción también evidencia una debilidad por no acabar las tareas $(\mathrm{X}=3.40 ; \mathrm{s}=0.548)$, sobre todo si existen complicaciones $(X=3.20 ; \mathrm{s}=1.095)$ y están poco seguras de la meta que se han propuesto $(X=3.20 ; \mathrm{s}=0.447)$. De igual manera, se consideran poco disciplinadas para lograrlo $(\mathrm{X}=3.00 ; \mathrm{s}=0.707)$.

En el caso de los alumnos de onceavo semestre, se encontró una puntuación de las medias en los ítems que evidencian que los estudiantes tienden a dejar sus metas $(\mathrm{X}=3.63$; $\mathrm{s}=$ 0.707) y experimentan la falta de disciplina $(X=3.63 ; \mathrm{s}=0.704)$; sin embargo, contrasta con su percepción de ser personas orientadas a las metas $(\mathrm{X}=4.25 ; \mathrm{s}=0.707)$ y resueltos en las metas que se proponen $(\mathrm{X}=4.38 ; \mathrm{s}=0.744)$. En las particularidades, el género masculino se caracteriza a sí mismo como muy trabajador $(X=5.00 ; \mathrm{s}=0.00)$, resuelto en las cosas elegidas, que nunca se da por vencido y acaba a pesar de los obstáculos $(X=4.75 ; \mathrm{s}=0.500)$.

\section{Discusión}

Los resultados obtenidos en el inventario VIA-240, indican que los estudiantes tienen un alto nivel de la Fortaleza del Carácter denominada Persistencia, lo cual indica que tienen 
tendencia a continuar voluntariamente su comportamiento de estudiar, pese a que enfrentan varios obstáculos como falta de descanso, viajes largos y los periodos largos y discontinuos de la carrera, que a la vez crean otras dificultades y problemas familiares por la falta de tiempo y en el aspecto psicológico relacionado al retardo de gratificación por lograr la meta.

En este caso, la licenciatura por 6 años pudiese ser un factor importante; sin embargo, con su Fortaleza de Carácter, a pesar de sus desalientos, buscan terminar lo que iniciaron, lo que exige una gran disposición psicológica, y evidencia a la vez que ellos, al menos durante este estudio, la han demostrado como elemento inherente a sus personalidades.

Este primer perfil descriptivo construido a partir de los resultados obtenidos en este estudio, sobre el nivel de Persistencia que presentan los estudiantes de Licenciatura en Educación Secundaria, con Especialidad en Formación Cívica y Ética, en modalidad mixta, del Centro de Actualización del Magisterio, representa en sí mismo un insumo fundamental para determinar el nivel de presencia de las 24 Fortalezas de Carácter que contempla el VIA-240, lo que a su vez nos permitirá a los docentes, implementar mejores estrategias de intervención en las aulas.

\section{Bibliografía}

Calderón, D., Forés, A., \& Gustems, J. (2016). Aproximación a las fortalezas de carácter en los estudiantes de Educación Social. Perfil de una muestra. REIRE, 9(2), 48-64. Recuperado el 12 de mayo de 2017, de 10.1344/reire2016.9.2924

Calderón, D., Gustems, J., \& Forés, A. (2016). El VIA-IS. Una herramienta para evaluar la eficacia de los planes docentes en los estudios de Educación Social de la Universitat de Barcelona. Revista CIDUI (3), 1-6. Recuperado el 13 de mayo de 2017, de http://www.cidui.org/revistacidui/index.php/cidui/article/view/1034/998

Cosentino, A. (2010). Evaluación de las Virtudes y Fortalezas Humanas en Población de Habla Hispana. Psicodebate, 53-72. Recuperado el 13 de mayo de 2017, de https://dspace.palermo.edu/ojs/index.php/psicodebate/issue/view/Psicodebate\%2010

Contreras, F., \& Esguerra, G. (2006). Psicología positiva: una nueva perspectiva en psicología. Diversitas, 2(2), 311-319. Recuperado el 04 de enero de 2018, de http://pepsic.bvsalud.org/scielo.php?script=sci_arttext\&pid=S179499982006000200011\&lng=pt\&tlng=es

IBM Corp. (2013). IBM SPSS Statistics for Windows, Version 22.0. Armonk, NY: IBM Corp.

Martín-Moreno, S. (2015). Fortalezas personales y rendimiento académico en estudiantes universitarios. Universidad de Jaén, Psicología. Jaén: Facultad de Humanidades y Ciencias de la Educación. Recuperado el 13 de mayo de 2017, de http://tauja.ujaen.es/handle/10953.1/1948

Peterson, Christopher \& Stephens, John \& Lee, Fiona \& Seligman, Martin \& Park, Nansook. (2009). Strengths of Character and Work. Oxford Handbook of Positive Psychology at Work. 221-231. 10.1093/oxfordhb/9780195335446.001.0001. 
Porto, N., \& da Fonseca, D. (2016). Asociaciones entre fortalezas del carácter y satisfacción con la vida: un estudio con universitarios. Acta Colombiana de Psicología, 19 (2), 90-96.

Park, N., Peterson, C., y Seligman, M. E. P. (2004). Character strengths and well-being. Journal of Social and Clinical Psychology, 23, 603-619.

Park, N., Peterson, C., y Seligman, M.E.P. (2006). Character strengths in fifty-four nations and the fifty US states. Journal of Positive Psychology, 1, 118-129.

Real Academia Española (2015) Perseverar. Real Academia Española. Recuperado el 15 de mayo de 2017, de http://dle.rae.es/?id=SjCGuM3

Saldaña, O., Escartin, E., Martin, J., Jiménez, Y., Ceja, L., Varela, A., Vidal, T., \& Rodríguez, A. (2014). Fortalezas personales relacionadas con el rendimiento académico y profesional en el campo de la Psicología Social y la Psicología Social Aplicada. I Congrés Internacional d'Educació Emocional i X Jornades d'Educació Emocional: Psicologia positiva i benestar. Institut de Ciències de l'Educació, Barcelona.

Seligman, M. E. P., \& Csikszentmihalyi, M. (2000). Positive psychology: An introduction. American Psychologist, 55, 5-14.

VIA Institute on Character (2017) Character Strengths. VIA Institute on Character. Recuperado el 15 de mayo de 2017, de http://www.viacharacter.org/www/Character-Strengths

VIA Institute on Character (2017) Perseverance. VIA Institute on Character. Recuperado el 15 de mayo de 2017, de http://www.viacharacter.org/www/Character-Strengths/Perseverance 